\title{
VIII.
}

\section{Zur Lehre von Urteil und Verneinung bei Aristoteles.}

Von

\section{Dr. phil. Willi Lewinsohn.}

Was ist für ảie Klärung des Begriffs der Verneinung, der bis in unsere Zeit hinein eine so bedeutende und vielumstrittene Rolle in der Theorie des Urteils spielt, bereits im ersten Aufriß der logischen Wissenschaften geschehen?

Vernein ung steht von Anfang an in einem besonders verwickelten Verhältnis zum Begriff des G e ge ns a t z es. Eine kurze Übersicht der Entwickelung dieses Begriffsverhältnisses bei den Vorsokratikern, bei Plato, endlich bei dem Schöpfer des Organon selbst, soll daher die Beantwortung unserer Hauptfrage vorbereiten.

Gegensatz und Verneinung sind ungetrennt und eines für die E l e a t e n. Daß ein Gegensatzglied die Verneinung, Aufhebung des anderen schlechthin sei, ist eine Grundüberzeugung des Parmenides und eine Grundvoraussetzung des Zeno ${ }^{1}$ ).

Umgekehrt, weil jedes Hinausgehen über „,das Eine“ notwendig zu einer Vielheit führt, d. h. zum „Gegenteil" des Einen, so wird es zur unausgesprochenen, aber dialektisch maßgebenden Norm bei ihnen, alles Nicht- $\boldsymbol{A}$ als „Gegenteil von $\boldsymbol{A}^{\text {" }}$ auszulegen. -

Gegènsatz und Verneinung fallen völlig auseinander bei $\mathrm{H}$ e $\mathrm{r}$ a kleitos. Die Gegensätze sind in Wahrheit Eins. Wenn also von zwei konträren Gegenteilen $m$ und $n$ (z. B. warm und kalt) dem

$\left.{ }^{1}\right)$ Úber die Rolle, die diese Voraussetzungen in Zenos Beweisen gegen Vielheit und Bewegung, wie auch in anderen eleatischen Argumentationen spielt, vgl. d. Verf. Dissert. über ,Gegensatz und Verneinung“ (Berlin 1910), S. $14 \mathrm{ff}$. 
$\boldsymbol{A}$ eines, m, zukommt, b raucht $\mathrm{nicht} \mathrm{n}$ von ihm verneint $\mathrm{zu}$ werd en.

Die seltsame Folge ist, daß Gorgias, der in seiner Logik dem Zeno folgt, zur Verneinung all er Urteil e gelangt; Protagmras, der von Herakleitos abhängt, zur Bejahung aller Crteile.

$\mathrm{Plat}$ ons Grundlehre geht von einer eleatisch gerichteten Behandlung von Gegensatz und Verneinung aus. Daß das, was z. B. weiß ist, $\mathrm{n}$ i $\mathrm{cht}$ und niemals schwarz sein könne, gilt ihm so absolut, wie die Ausschließung von Einheit und Vielheit für Zeno unbedingt galt; darum ist durch ihr $\mathrm{S} c \mathrm{~h}$ wa $\mathrm{nken} \mathrm{z} w$ ischen Gegensätzen die sichtbare Welt, gegenüber der Ideenwelt, gezeichnet. Wie der der $\mathrm{Un}$ wahrheit überführt wäre, der mit Protagoras denselben Sachverhalt bejahen und verneinen wollte, so sind die Sinnen-Dinge ihrer Unwirklichkeit überführt, weil in ihnen alles mit seinem eigenen Gegenteile behaftet auftritt: kein Gerechtes, das nicht auch ungerecht wäre, usw. (vgl. Rep. 479 a usw.)

Bei den von den sophistischen Schulen in den Dingen überall aufgewiesenen Widersprüchen beruhigt sich nun Platon im Hinblick auf die reinen Urbilder, die selber von ihren Gegensätzen völlig unbefleckt bleiben, und an denen jene Dinge bloß ,teilhaben“"

Dabei ergeben sich Schwierigkeiten, die Platon zu einer Modifikation des eleatischen, ja auch des eigenen ursprünglichen Standpunktes hindrängen.

Einerseits kann vom Zeitlich-Räumlichen nicht soweit abstrahiert werden, daß die Ausschließung des (für unsere Begriffe) Kontradiktorischen nicht auch für die Sinnendinge gefordert werden müßte: auf diese bezieht sich bereits der Satz, daß ,dasselbe nicht zugleich und in derselben Hinsicht dem Nämlichen (resp. vom Nämlichen) Entgegengesetztes tun (resp. leiden)" könne. (Rep. IV, 436 b.)

Anderseits kann in der Sphäre der Ideen selber eine. absolute gegenseitige Abstoßung und Ausschließung aller $\dot{\varepsilon} \nu \alpha \nu \tau i \alpha$ nicht gedacht werden, ohne daß wir der Parmenideischen Konsequenz des starren All-Eins anheimfallen. Solange die $\varepsilon^{\prime \prime} \delta \eta \eta$ der Einheit und Vielheit, der Identität und Verschiedenheit als sich gegenseitig vollkommen ausschließend verstanden werden, läßt sich die Einheit zweier Begriffe, mithin die Möglichkeit des Urteils, die Möglich- 
keit einer Gemeinschaft unter den Ideen überhaupt nicht begreifen. Damit es eine Wissenschaft von diesen gebe, mü s s e n mithin gewisse Gegensätze vereinbar sein für die Vernunft, nicht bloß für die Sinne: so Einheit und Vielheit, Identität und Verschiedenheit; Grenze und Unbegrenztes ${ }^{2}$ ).

Die Urteilseinheit schwebt Platon als Forderung vor; völlig realisierbar ist sie innerhalb seines Systems nicht.

, $\boldsymbol{A}$ is t $\boldsymbol{B}^{\prime \prime}$ bedeutet: $(\boldsymbol{\alpha})$ die Idee $\boldsymbol{A}$ hat teil an der Idee $\boldsymbol{B}$, aber auch: $(\boldsymbol{\beta}) \boldsymbol{A}$ und $\boldsymbol{B}$ haben teil am Sein, an der Idee des Seins ${ }^{3}$ ).

, $\boldsymbol{A}$ is t nicht $\boldsymbol{B}^{\prime \prime}: \boldsymbol{A}$ und $\boldsymbol{B}$ haben teil am Nichtsein ${ }^{4}$ ). Dieses Nichtsein definiert Platon als gleichbedeutend mit ,Verschiedensein“. $\boldsymbol{A}$ kann nun aber ersichtlich von $\boldsymbol{B}$ v e r s c h i ed e n sein, während gleichwohl beide, einzeln sowie zusammen, teil haben am $\mathrm{Sein}$; dann gilt $\boldsymbol{A}$ ist $\boldsymbol{B}$ und $\boldsymbol{A}$ ist nicht $\boldsymbol{B}$ : Bejahung und Verneinung des Nämlichen sind, der logischen Form nach, für ihn ebensowenig unvereinbar, wie sie es, der realen Geltung nach, für Protagoras waren.

Der Begriff des Kontradiktorischen ist so bei Platon noch nicht entwickelt. Sein Ėvaviíov bleibt immer noch der populäre Sammelname der „Gegenteile" wie: groß und klein, gerecht und ungerecht. bewegtsein und ruhen. Als ihre logische Charakteristik ist im wẹsentlichen die der gegenseitigen Ausschließung schlechthin festgehalten. Fast nur gelegentlich, weil nur für die Sinnenwelt belangvoll, findet sich jene präzise Bestimmung über Ausschließung der čvaviia, s of e r n sie and e m selben Gegenstand in id e n t is cher $\mathrm{Zeit}$ und $\mathrm{Hinsicht}$ auftretend gedacht werden. Aber auch

2) Das gilt genau genommen für alle Gegensätze, die eben (nach dem „Sophistes") mit dialektischer Rücksicht auf die Mannigfaltigkeit der Be. griffsbeziehungen aufzufassen sind: Die Ideen der Ruhe und der Bewegung haben gemeinsam an der des "Seins", und insofern aneinander teil. - Vgl. die Diss. S. $34 \mathrm{ff}$.

3) Jedes einzeln (existenziales Sein); und auch beide zusammen (als Urteilsmaterie; - modales Sein; entspricht der Deutung $\alpha$ ). Plato weiß darin keinen Unterschied.

4) Wiederum jedes auch für sich. - Mit der Hypostasierung der Funktionsbegriffe zu I dee n der Identität und Verschiedenheit, an denen die Urteilsglieder "Teil haben“, ist die Erfassung der logischen Kopula ausgeschlossen. 
hier bezieht sich der Gedanke der Repugnanz auf die Prädikatsbegriffe, die als solche auf Grund begrifflicher, in haltlicher Bestimmtheiten einander ausschließen. Über diese hinaus zum f ormalen Gegenüberliegen zweier Urteile in Bejahung und Verneinung, mithin zu einem logisch völlig faßbaren Begriff der contradictio, gibt es für Platons Begriffsphilosophie keinen Weg. -

Aristoteles unterscheidet unter den Bedeutungen des

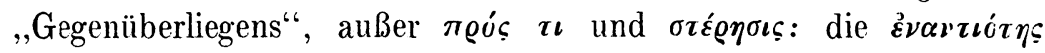
und die ảvií $\alpha \sigma \iota \varsigma$.

Hier erhält Platons ċrcuriov seine bestimmte Aufklärung:

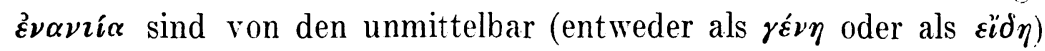
vergleichbaren, ,unterschiedenen“ Begriffen diejenigen, deren $A b$ stand der größte, d. h. deren Unterschied vollendet ist ${ }^{5}$ ). Damit

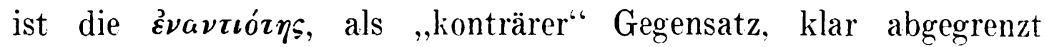

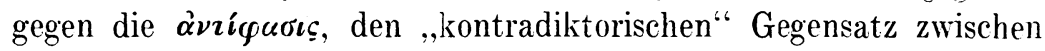
zwei Sachverhalten oder Urteilen, laut denen das Nämliche vom Nämlichen gilt oder nicht gilt, bejaht oder verneint wird. Der Begriff der Antiphasis ist vollkommen entfaltet in zwei Axiomen: dem Satz des Widerspruchs (Met. $\Gamma 3,1005$ b 19), und dem auf Platos Standpunkt noch überhaupt nicht begründbaren Satz vom ausgeschlossenen Mittleren ( $\Gamma 7,1011$ b 23 ff.).

Von den beiden logisch verschiedenen Momenten, die in der

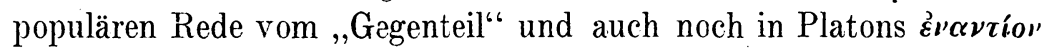
ungesondert auftraten, der Unverei n b a rke it und der U n t e r schiedsgröße, ist jetzt jene als das Wesen der ảvticforıৎ,

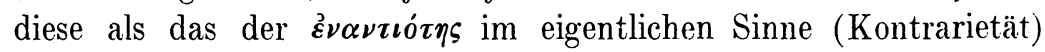
herausgestellt. -

Wo in der klaren Abgrenzung zwischen Begriff und Urteil bei dem großen Schüler Platons eine Lücke geblieben ist, da erhebt sich der Begriff der Privation. Unter seinen verschiedenen Schwankungen ist wichtig, daß von Privation geredet wird einmal: wo einem Dinge etwas fehlt, das ihm von Natur zukäme, - oder aber, wo ihm schlechthin etwas abzusprechen ist; ferner: wo einem Dinge etwas völlig abzusprechen, - oder aber ihm bloß in geringerem Maße,

5) Vgl. bes. Met. $\iota 4,1055$ a $3 \mathrm{ff}$.; dazu $\iota 3$ 1054b $25 \mathrm{f}$., wo das ,Unter-

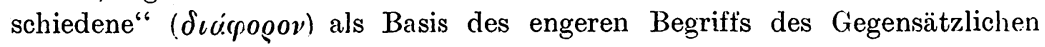
erläutert ist. - Vgl, a. die Dissert. S. 62-65. 
als ihm zukäme, zuzusprechen ist. Mit dieser letzten Bestimmung

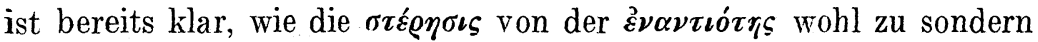
ist: in jeder Stufenreihe können nur die Extreme in konträrem, dagegen sowohl diese als auch alle $\mathrm{Mit}$ t els t u f e $\mathrm{n}$ in steretischem Verhältnis zueinander stehen. Der ersteren Alternative gemäß aber erscheint, sobald die Privation an das bestimmte Ding nicht

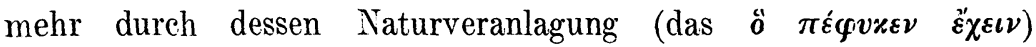
gebunden ist, Haben und Nichthaben oder Entblößtsein lediglich als das auf ein bestimmtes Substrat (ixoxeíusvov) gleichsam projizierte Sein und Nichtsein der Aussage, als real gefaßte Bejahung und Verneinung. - In concreto bezeichnet hingegen die als Realattribut auftretende Schein-Negation stets eine dem fraglichen Sub-

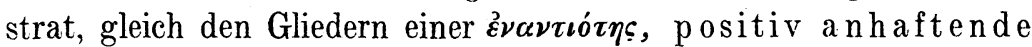
Inhärenz ${ }^{6}$ ). Demgemäß finden sich nun erstlich Verneinung und Gegensatz vielfach einander näher gerückt, als es den Grundbegriffen beider entspräche: durch bloße steretische Verneinung des einen $\varepsilon^{\prime}{ }^{\prime} \alpha \nu i_{i o v}$ erscheint das andere erzeugt ${ }^{7}$ ), gleichwie sprachlich, durch bloße Zufügung der Privations-Silbe, der nämliche Wortstamm dem Áusdruck des entgegengesetzten Tatbestandes dienstbar wird; zweitens der Privationsbegriff rückwirkend als Stütze einer Urteilsauffassung, die der Negation ein reales und positives Fundament unterzubauen strebt.

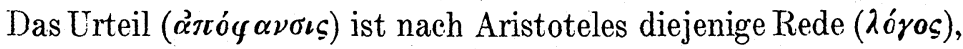
der ein Wahr- oder Falschsein zukommt (de int. 17 a 2). Dieser Rede als einem Zusammenschluß zweier Lautgebilde, entspricht in der Seele ein Gedanke, der seinerseits dadurch, daß er notwendig ,wahr oder falsch" ist ${ }^{8}$ ), von dem durch das einzelne Wort repräsentierten Gedanken unterschieden" wird (a 10). Im Realen aber entspricht ihr ein $\pi \varrho \tilde{\alpha} \gamma \mu \alpha$ im eigentlichen Sinne, d. h. etwas Faktisches,

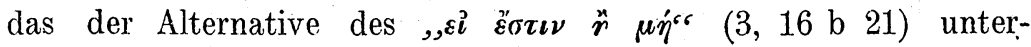
liegt $\left.{ }^{9}\right)$.

6) z. B. „Unfreundlichkeit“ eines Menschen bald Feindlichkeit, bald irgendeine Stufe der Annäherung an diese.

$\left.{ }^{7}\right)$ Met. $\Theta 2$; vgl. Dissert. S. 75 f.

${ }^{8)}$ de int. 1. 16a 9-11.

$\left.{ }^{9}\right)$ Dagegen wird irgendein Gegenstand, ein $\pi \varrho \tilde{a} \gamma \mu \omega$ im weiteren Sinne, (vgl. a. Maier, ,Syllogistik des Aristoteles“, Bd. I, S. 115, 3) - ohne Setzung oder Verwerfung seiner Existenz - auch dem isolierten Wort und seinem 
Im Urteil kann, da dieses Faktische im ersten Falle (des siva $)$

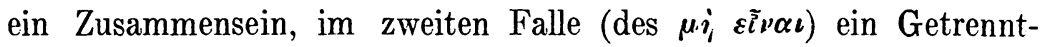
sein bedeutet (Met. $\Theta 10.1051$ b 11 f.), nun entweder auf ein ob-

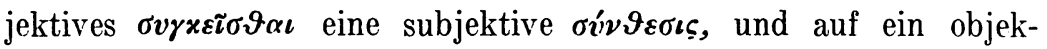

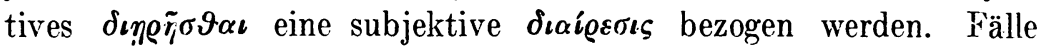
wahrer Bejahung und Verneinung. - Oder es kann auch das umgekehrte Beziehen eintreten. Fälle falscher Bejahung und falscher Verneinung (ibd. b 2-5). -

Die Alternative von Falsch und $W a r^{10}$ ) hat stets eine $\sigma v \mu \pi \lambda \alpha_{0} y$ zur Voraussetzung (so bereits Cat. 4, 2 a $6-10$ ). W a h r h e it aber reicht über das Gebiet der dianoetischen Verbindungen und Trennungen, mithin überhaupt das des Urteilens, hinaus. Wie, mit gewissen Einsehränkungen, die spezifischen Sinneswahrnehmungen (De an. III, 3, 427 b 12; vgl. 428 a 11; 430 b 29), so ist auch das intuitive Denken des unteilbaren Wesensbegriffes, und der einfachen Substanzen - einfach wahr. Es hat sein, - dem $\psi \varepsilon \tilde{i} \delta \delta \varsigma$ der dianoetischen Sphäre etwa entsprechendes, - Gegenteil nicht in einem Falschdenken, sondern höchstens in einer Nichtbetätigung des Denkens ${ }^{11}$ ).

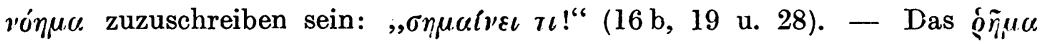
„Sein", für sich genommen, ist auch hierin allen anderen isolierten Worten gleich. Auch jenes ,bedeutet etwas“ (dazu M a i e r S. 114 f). Weil aber die Versuchung nahe liegt, bei ihm, dessen I n h alt auf die Tatsächlichkeit selber geht, darum die für jede Setzung einer Tatsächlichkeit erforderliche F o r m, nämlich die urteilsmäßige Synthese, für irrelevant zu halten, deshalb nur hebt Arist. hier (16b $22 \mathrm{ff}$.) den Unterschied zwischen logischem

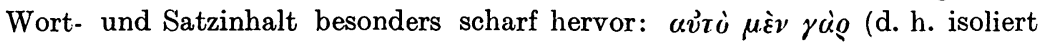

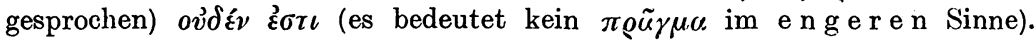
- Sobald man diese Worte strenger versteht, widersprechen sie der Vor-

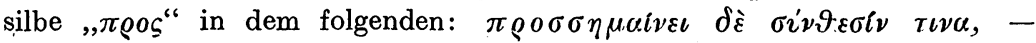
und aller sonstigen Behandlung des Seins. Das hat $\mathrm{S} t \mathrm{e}$ in th a $\mathrm{l}$ (Geschichte d. Sprachw. bei Gr. u. R., 2. Aufl., I, S. 241 f.) mit Recht betont. Doch

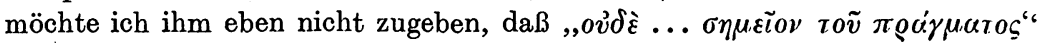
vom "Sein" in anderer Bedeutung gelte, als von irgendeinem sonstigen isolierten Worte. Ich verstehe die Stelle nicht gleich ihm dahin: „das E sei kein Stoffwort".

10) $\mathrm{Zu}$ beachten ist sogleich die Betonung des $\psi \varepsilon \tilde{v} \delta o \varsigma$; so De an. III, 6,430 b 2; vgl. 430 a 27 .

11) Met. $\Theta 10,1051$ b 32; b 25. - Vgl. $E 4,1027$ b 27. Dazu M a i e r, I, S. 7 , S. 19 f. - Prantl; S. $114 \mathrm{f}$. 
Das reine, begriffliche Erfassen des $\boldsymbol{\imath} \hat{\imath} \boldsymbol{z} \sigma \boldsymbol{\imath \iota \nu}$ erscheint also zwar gleichfalls logisch bewertbar; - doch nur in affirmativer Richtung: als "Wahrheit". - Und daß der Wahrheitsbegriff sich hierher erstreckt, hat nun eine über das Terminologische weit hinausreichende Bedeutung. -

Der Wesensbegriff enthält nämlich, wie nach der metaphysischen Seite den Seins-Grund, so nach der logischen Seite das „Ausgesagtwerden" von dem, was unter ihn fällt, eigentlich unmittelbar in sich. Dies ergibt im Anfang eine logische Unsicherheit im Yerhältnis von Begriff und Urteil. Die Schwierigkeiten zentrieren um den Begriff der $x \alpha \tau \eta$ rogi $\alpha$. Nach Steinthals ${ }^{12}$ ) überzeugender Darlegung bezeichnet diese ,,allerdings Prädizierung, Aussagen eines Etwas von Etwas; d. h. aber bei Aristoteles ursprünglich: Aussagen eines Wortes als eines bestimmten Begriffes, ohne Beziehung auf seine Stellung im Urteil, aber mit Beziehung auf die im Worte gedachte Sache, von der es prädiziert wird; also das Wort als Prädikat des Dinges ist $\boldsymbol{\alpha} \alpha \tau \eta$ rói $\alpha . "$

Die - in diesem Sinne prädizierbaren - isolierten Worte

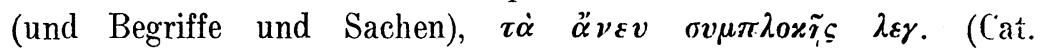
$1 \mathrm{a}, 17 \mathrm{f}$.) sind es, für die in der bekannten Einteilung (Cat. Cap. 2) das $\varkappa \alpha Y^{\prime}$ i் $\pi$ $\lambda \varepsilon \gamma \varepsilon \sigma \vartheta \alpha \iota$ zum fundamentalen Einteilungsgrunde wird.

Die Kategorienschrift kennt nun aber ersichtlich nur e in e

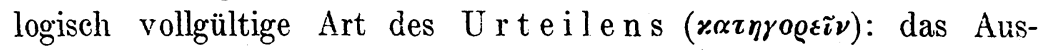
sagen der zweiten Substanzen (inkl. Artdifferenzen) von den ersten. Denn nur dabei wird der Begriff, mit dem Worte zugleich, prädiziert. ${ }^{13}$ )

12) 1. c. S. 208. Zum ganzen Abschnitt vgl. S. 206-35. - Zum Kategorienbegriff ferner Trendele $\mathrm{nburg}, \mathrm{p} .3 \mathrm{ff}$. Bonitz, Ind. Arist. p. 377. W a itz, Org. I, 266 ff. M a i e r II, 2, S. 290 ff. - Zur Frage der Echtheit der Kat.-Schrift ibid. S. 291, 1.

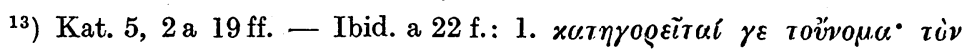

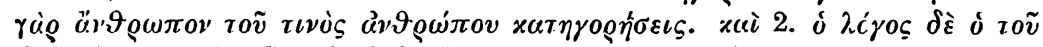
¿้ๆ

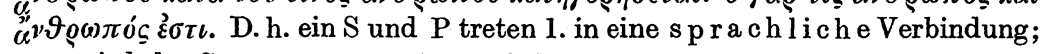
man wird den Satz a us sprech en: Sokrates ist Mensch; - hier aber außerdem noch: 2 in ein begriffliches Verhältnis. „Mensch ist der Wesensbegriff, worin "Sokrates" logisch enthalten ist. - Eben dies macht „Mensch“ 
Hier spielt mithin jene Bedeutung von xatryogi $\alpha$, wonach diese eine „Aussage eines begrifflichen Inhalts im Worte" ist (so Maier II, 2, 304, 1), eine wichtige Rolle für die Auffassung des Wesens der Urteils funktion: noch ist nicht die Alternative von Verbindung und Trennung ihr als wesentlich zuerkannt; statt dessen dieses,

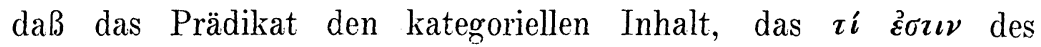
Subjektes auf Grund eines begrifflichen Umfangsverhältnisses darstellt $\left.{ }^{14}\right)$.

Dies stimmt mit der maßgebenden Behandlung des Lrteils durch Aristoteles (Hermen., Analyt.) ersichtlich nicht überein. und so sind wir wohl einstweilen berechtigt zu der durch die herrschende Datie-

zu einer жutqrogí des Dinges (Sokrates) im oben bezeichneten Sinne. Wird dagegen ein P (z. B. ,weiß") mit einem S (z. B. Sokrates) rerbunden,

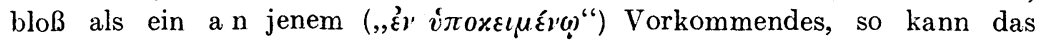
nicht als eine Aussage des Begriffes, sondern bloß des Wortes gelten (2 a 27-34), ist also eine logisch auf dieser Stufe der Theorie noch überhaupt nicht $\mathrm{zu}$ rechtfertigende Prädikation (vgl. ibid. IIIa 15-28). Dazu vgl. Steinthal, $217 \mathrm{ff}$.

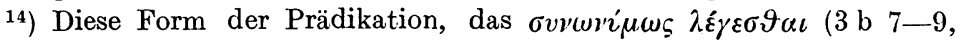
vgl. 3 a 33 ff.), wird den zweiten Substanzen und den Artdifferenzen zugeschrieben; - wozu also nur ovoícı wiederum das Subjekt bilden. Die Kateg. selber vernachlässigen das, was , „uף’

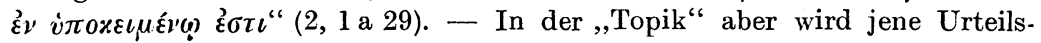
form sämtlichen höchsten Gattungen - für Subjekte der jeweilig nämlichen Gattung - zugesprochen. Ist das (als Subjekt) Daliegende selber eine Qualität, so kann nur eine Qualität, z. B. „weiß“ (als allgemeiner Begriff) hierfür das

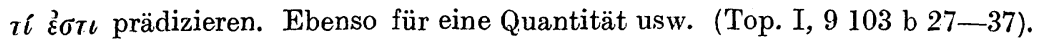
- Liegen $\mathrm{S}$ und $\mathrm{P} \mathrm{nich} \mathrm{t}$ in der nämlichen kategorischen Gattung (ö $\tau \alpha \nu$

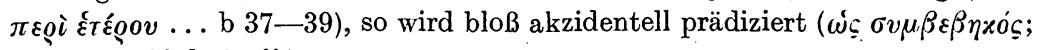
s. IV, 1120 b 21 ff.). -

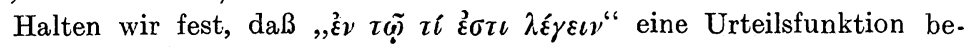
deutet (freilich nicht mehr die Urteilsfunktion schlechthin, wie in Kat.), so scheint mir deren Behandlung in I, 9 durchaus nicht widerspruchsvoll (wie nach Steinthal, S. 222). Bei Gattungsgleichheit des S und P kann das

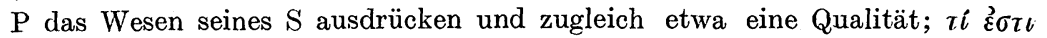

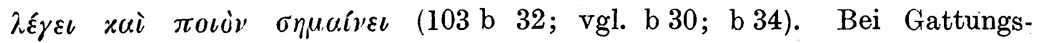
verschiedenheit aber kann es, mit veränderter Urteilsfunktion, offenbar nicht mehr das Wesen des S ausdrücken; sondern bloß noch etwa eine Qualität,

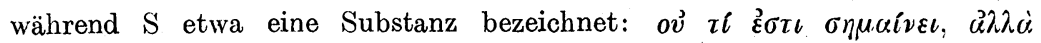

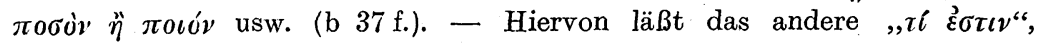
das in $103 \mathrm{~b} 22$ als I $\mathrm{nhalts}$ - Bestimmtheit und Kategoriengattung auftritt, sich immer noch deutlich scheiden (vgl. auch Waitz II S. 447. - Anders freilich Maier l. c. S. 321. 
rung der genannten Schriften nahegelegten Annahme, es handle sich dabei um eine f $r$ ü h e r e Stufe der Theorie.

Es erhellt nun, daß für diese ältere Anschauung vom logischen Wesen des $x \alpha$ i $\eta$ rogזĩ nur allenfalls negative Inhalte des Denkens sich ergeben mochten - soweit nämlich Privationen, als die eine Richtung der Inhaltsbestimmtheit selbst, und daher

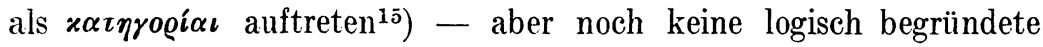
negative Urteilsform: Der Forderung, daß nicht bloß das Wort, sondern ( $\vec{\varepsilon} v \boldsymbol{\tau}(\hat{i}) \tau i \vec{\varepsilon} \sigma \tau \iota)$ zugleich auch der Begriff prädiziert werden solle, können nur Aussagen genügen, deren Prädikat das $S \mathrm{u} \mathrm{b} \mathrm{j} \mathrm{e} \mathrm{k} \mathrm{t} \mathrm{w} \mathrm{i} \mathrm{r} \mathrm{k-}$

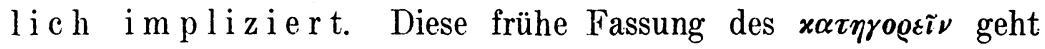
mithin parallel mit der auch später beibehaltenen Lehre vom intuitiven Denken (voi's): sofern dieses überhaupt nur da statthat, wo der Gegenstand wirklich e r f a B wird (0. S. 202) ${ }^{16}$ ).

Diese Stufe der Urteilstheorie zeigt uns aber die Brücke zwischen Plato und dem Standpunkte der Hermenie. Wenn für Platos Bewußtsein ein Widerspruch (in Gestalt seiner $\dot{\varepsilon} \nu \alpha \nu \tau \iota$ ó $\eta \varsigma$ ) nur zwischen gewissen Begriffen - und ihnen korrespondierenden Dingen - obwaltet, weil diese, aus nicht aufgeklärtem Grunde, $\ddot{\alpha} \lambda \lambda \eta \eta \alpha$ ov $\delta \dot{\varepsilon} \chi o \nu \tau \alpha \iota$, - so besteht für den reifen Aristoteles hingegen reine contradictio in letzter Linie zwischen Urteilen, resp. den ihnen entsprechenden realen Sachverhalten, die einander als Verbundensein eines $A B$, und Getrenntsein eines A B, gegenüberliegen. Auch hier behält

15) Hierzu Phys. III, 1201 a 3-8. Es spielt hier wiederum jene scheinbare Einheit von Sterese und realem Gegensatz herein (o. S. 201). Vgl: auch Prantl, Geschichte der Logik I, S. $193 \mathrm{f}$.

16) Daher findet die logische Alternative von Bejahung und Verneinung in dem echten Teile der Kateg.-Schrift gar keinen Raum. Dies ist sehr bedeutsam vor allem im Kap. 4, wo vom Urteil ausdrücklich die Rede ist:

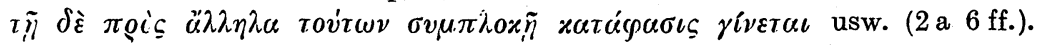

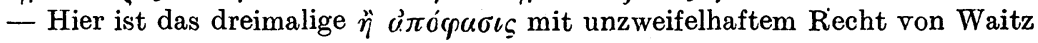
(s. I 281) als späte Interpolation gestrichen worden.

Von hier aus aber begreifen wir auch spätere Äußerungen über die Verneinung, wie die bekannte Stelle Anal. post 25, $86 \mathrm{~b} 33 \mathrm{ff}$. - Und wenn in

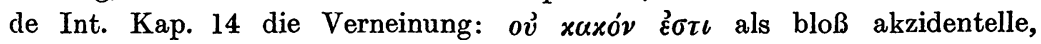
dagegen das entsprechende positive Prädikat als wesentliche Aussage über das "Gute" erscheint ( 23 b 15 ff.), wofür die Herm. selbst keinerlei Begründung enthält, so sind wir damit auf Kat. und Top. zurückverwiesen.

Archiv füx Geschichte der Philosophie. XXIV. 2.

14 
nun die Verneinung noch einen stark positiven Charakter insofern, als der negative Satz gleich dem affirmativen, lediglich ein objektiv Vorliegendes (den Sachverhalt der Trennung) zu formulieren hat. Allein gerade vermöge des Gedankens von Verknüpfung und Trennung ist die Urteilstheorie jetzt soweit entwickelt, daß die Urteilsform sich loslöst von den bloßen Verhältnissen - und implicite-Aussagen

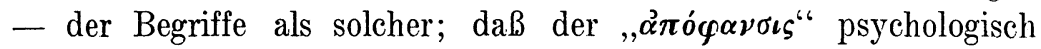

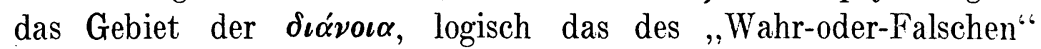
zugewiesen, damit zugleich aber die formale Koordination von Bejahung und Verneinung geschaffen werden kann. Konnte bei Plato

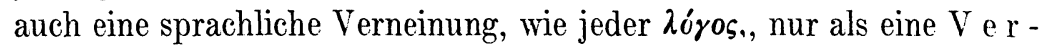
b in d u n g begriffen werden, nämlich als Gemeinschaft der Urteilsglieder mit dem Nichtsein oder Verschiedensein, so ist nunmehr hingegen die Negation (als Abbild des Getrennt-Seienden) in der Urteilsfunktion selber von der Affirmation geschieden, und neben diese gerückt. G'eichwohl erkennen wir die Nachwirkung der platonischen Begriffslogik nach zwei Seiten hin deutlich: Einmal war in der frühen Kat.-Schrift von jener sicheren Erfassung der Bejahung und Verneinung noch keine Rede, vielmehr fanden wir dort ganz ähnlich wie bei Plato nur: 1. isolierte Begriffe (resp. Worte Sachen).

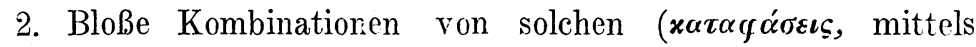
$\left.\sigma v \mu \pi \lambda \sigma \% \eta^{17}\right)$.

Andererseits aber tendiert hierin der aristotelische Objektivismus dauernd zurück, sofern in seinen realen Diäresen die Teile schließlich

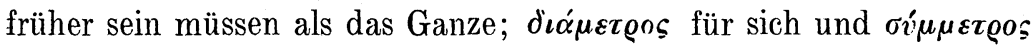
für sich, resp. in irgendwelchen Kombinationen mit anderem, irgendwie da sein müssen, u n a b h äng $1 \mathrm{~g}$ von ihrer realen, dem wahren negativen Urteile vorbildlichen Diärese ${ }^{18}$ ).

$\left.{ }^{17}\right)$ Auch die Feststellung, daß Wahr oder Falsch lediglich diese $x \alpha \tau \dot{\alpha}$.

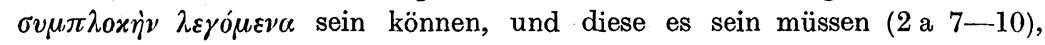
geht über Plato. (s. Soph. p. 262 f.) hier nicht hinaus.

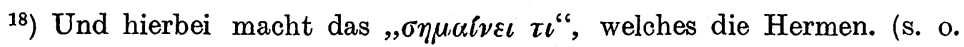
S. 11, Anm. 3) den e in z e l n e n Worten resp. Satzteilen zuspricht, zuletzt doch nur eine gewisse Verlegenheit deutlich darüber, welcher Realitätswert denn eigentlich dem, was diese objektiven Verbindungen und Trennungen e ingeht, zukommen mag. 
Im bejahenden Satze ist das sĩva ${ }^{19}$ ), im verneinenden das $\mu \dot{\eta}$

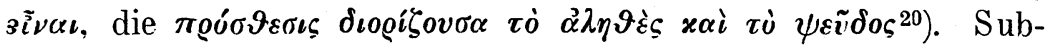
jekts- und Prädikatswort (wo nämlich außer dem हैorı noch ein weiteres

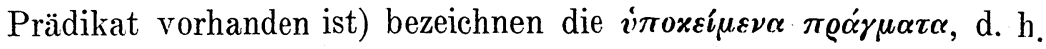

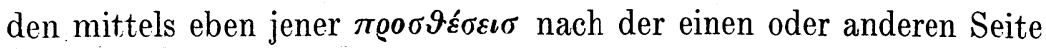
zu beurteilenden Inhalt.

Die Grundlage für das Verhältnis von Bejahung und Verneinung ist nun in der Weise gegeben: $x \alpha \tau \dot{\alpha} \varphi \alpha \kappa \iota \varsigma$ und $\dot{\alpha} \pi \dot{c} \varphi \alpha \sigma \iota \varsigma,, \tau o \tilde{v} \alpha \hat{\imath} \cdot \tau \tilde{v}$

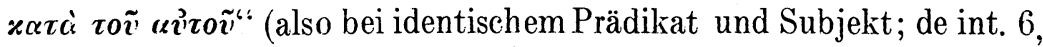
17a 35; vgl. 7, 17b 38), liegen einander als Antiphasis, nach dem Axiom also als das nicht zugleich Wahrsein-Könnende gegenüber. Und nur da werden wir von Verneinung eines Urteils eigentlich reden können, wo dies Verhältnis zur Bejahung vorliegt, daß, wenn diese wahr, notwendig jene falsch ist und umgekehrt. - Ist es daher stets statthaft,

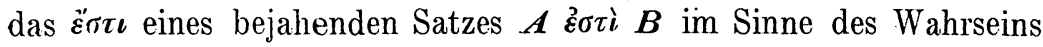

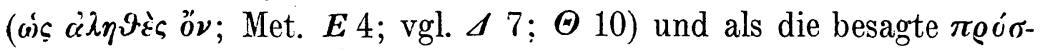
צैศıৎ aufzufassen, so ergibt es sich als gleichgültig und für Aristoteles unproblematisch, ob ich im gegenüberliegenden Satze $\boldsymbol{A}$ ov้» $\boldsymbol{\varepsilon} \sigma \tau \iota \boldsymbol{B}$

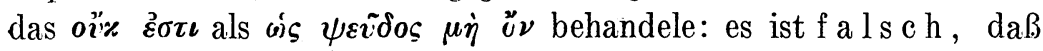
$A B$ sei, oder ob ich mich unmittelbar auf den Gegenstand beziehe, und ein reales Getrenntsein von $\boldsymbol{A}$ und $\boldsymbol{B}$ (mithin eine. Verneinung als wahr) konstatiere.

Außer der so festgelegten Satzverneinung bietet aber die Sprache noch andere Verwendungen des Negationszeichens dar.

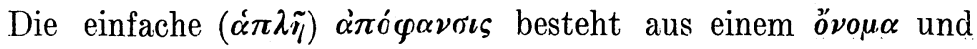
einem $£ \tilde{\eta} u \alpha$, welche nach der logischen Seite hin Subjekts- und Prädikatswert repräsentieren ${ }^{21}$ ). Beide sind entweder zu einer Bejahung

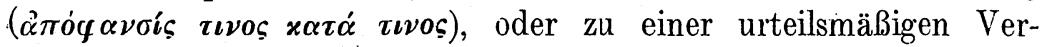

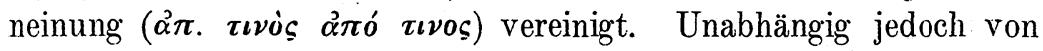
dieser kann die sprachliche Verneinung zunächst das övoux betreffen.

Ein solches negatives, „unbestimmtes“ övo $\mu \alpha$ kann nun außer der Subjektsstelle, wo es natürlich keine Negation des Satzes sein

19) Dieses ist aber auch in jedem anderen $\oint \tilde{\eta} \mu \alpha$ logisch enthalten und sprachlich an jedem darstellbar (De Int. 12, 21 b 6-10; vgl. 10, 20 a 3 ff. u. ö.).

$\left.{ }^{20}\right)$ l. c. 12,21 b 26-32. Dazu 21 b 20-22. Vgl. Maier I S. 111, 3, S. 113.

- Waitz, Organon I, 357.

21) S. darüber de Int. Cap. 2-3. Ferner 10, 19 b $10 \mathrm{f}$. 
kann (s. de int. 10, 19b 11-19), auch an der Stelle auftreten, die wir grammatisch als die des Prädikatsnomens bezeichnen ${ }^{22}$ ).

Bei dieser Verwendung fragt sich, ob als widerspruchsweise Verneinung des Urteils , $\boldsymbol{A}$ ist $\boldsymbol{B}^{\prime \prime}$ das , $\boldsymbol{A}$ ist Nicht- $\boldsymbol{B}^{\prime \prime}$ gelten dürfe. Mit anderen Worten: ob dieses mit dem Satze , $\boldsymbol{A}$ ist-nicht $\boldsymbol{B}^{\text {" }}$ logisch

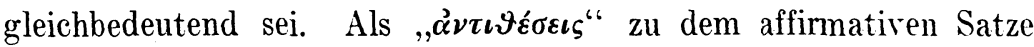
erkennt Aristoteles zwar Beides an (19 b 20); allein als Widerspruch im technischen Sinne durchaus nur das zweite. Die Verneinung des Satzes , $\boldsymbol{A}$ ist weiß" lautet nicht: ,, $\boldsymbol{A}$ ist nicht-wei $ß^{\prime \prime}$, sondern lediglich „A ist-nicht weiß" (An pr. I, 46, p. 51 b 7 ff; vgl. b 25 f.).

Den (51 b 10-25) hierfür gegebenen Beweis tadelt Wa it $z$ ( $(, 477)$ nicht ohne Grund; doch mit nicht ganz zutreffender Begrüindung. 1)ie Sätze, $\boldsymbol{A}$ ist $\boldsymbol{B}^{\prime \prime}$ und, $\boldsymbol{A}$ ist nicht- $\boldsymbol{B}^{\prime \prime}$, heißt es, verhalten sich gerade so wie , $\boldsymbol{A}$ kann $\boldsymbol{B}^{\prime \prime} \mathrm{zu}, \boldsymbol{A}$ kann nicht- $\boldsymbol{B}^{\prime \prime} !$ Wenn aber , $\boldsymbol{A}$ kann

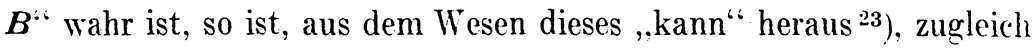
auch , $\boldsymbol{A}$ kann nicht- $\boldsymbol{B}$ " wahr. Dagegen ist nach dem Axiom unmöglich, daß mit dem , $\boldsymbol{A}$ kann $\boldsymbol{B}^{\prime \prime}$ zugleich das , $\boldsymbol{A}$ kann-nicht $\boldsymbol{B}^{*}$ wahr sei. Ein Vermögen des $\boldsymbol{A}$ in Richtung $\boldsymbol{B}$ kann unmöglich zugleich stattfinden und nicht stattfinden. - Folglich können die beiden

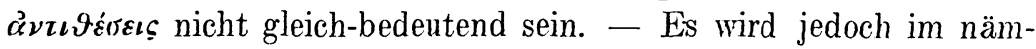
lichen Kapitel die Figur entworfen:

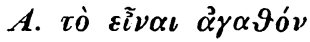

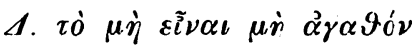

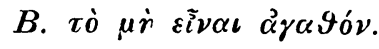

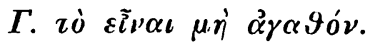

$\left.{ }^{22}\right)$ Soweit reicht die Kraft der zugleich sprachlichen und logischen Kategorien övouce und $\oint \tilde{\eta} \mu \alpha$, sowie die Tiefe dieser ganzen Analyse freilich nicht, um diese zwei Stellungen des övouc streng auseinander zu halten. - Hier sind sehr lehrreich Übergänge wie in $12,21^{\circ} \mathrm{b} 1-3 ; \mathrm{b} 7 \mathrm{ff}$, wo zum verneinten Prädikatsnomen das negative Subjektsnomen in enge Parallele gebracht ist. Gemäß der in b 9 f. gegebenen Anregung müßte der Satz b 7-8

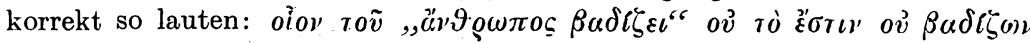

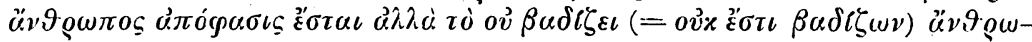
$\pi 0 \varsigma$ (dazu s. u. Anm. 27); ähnlich 10, 20 a 8 ff. - Das ganze Verfahren entspricht hierin völlig jenem Formalismus, mit dem in $21 \mathrm{~b} 10 \mathrm{ff}$. der den

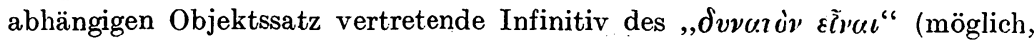
$\mathrm{z}$ u sein) mit dem nur den Hauptsatz vervollständigenden Infinitiv des $\lambda \varepsilon v \times \dot{\nu} \nu$

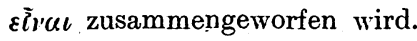

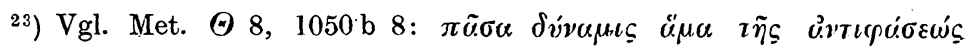

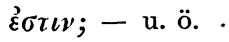


Jedwedem Substrat wird nun (51 b 39) entweder A zukommen oder $\boldsymbol{B}$, und keinem beides. Desgleichen entweder $\boldsymbol{\Gamma}$ oder $\boldsymbol{\Delta}$. Jedem aber, dem $\Gamma$ zukommt, m u $B$ auch $B$ zukommen, während hier nicht das Umgekehrte gilt (b 41-52 a 5).

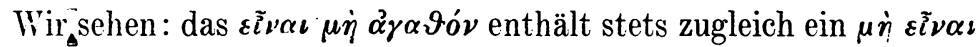

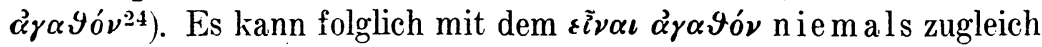
wahr sein. - Die ganze logische Form, wonach das , $\boldsymbol{A}$ kann $\boldsymbol{B}^{\text {" }}$ mit dem , $\boldsymbol{A}$ kamn nicht- $\boldsymbol{B}^{\prime \prime}$ zugleich wahr, ja dieses durch jenes gefordert ist, bleibt mithin dem $\left.\delta_{i}^{\prime} v \alpha \sigma \vartheta \alpha \iota^{25}\right)$ eigentümlich. Das , $\boldsymbol{A}$ ist weiß" verhält sich ersichtlich zu dem , $\boldsymbol{A}$ ist nicht-weiß" in völlig anderer Weise; und jene. Voraussetzung des $\delta \mu v^{i} \omega \varsigma \varepsilon^{\prime \prime} \chi \varepsilon \iota \nu$ (51 b $10 \mathrm{f}$.) erweist sich als ungültig. Damit fällt der Beweis. Das Bewiesene aber bleibt davon unberührt bestehen.

\section{Negation beim $\varrho \eta \mu \alpha$. - Das $\varrho \tilde{\imath} \mu \alpha \dot{\alpha} o ́ \varrho \iota \sigma o v$ und die Urteilsverneinung.}

Die sprachliche Verneinung heftet sich nun ferner an das $\varrho \tilde{q} \mu \alpha$. Betrachten wir das Urteil zunächst von seinen sprachlich ausgezeichneten Bestandteilen aus - dies aber ist die Methode, womit die Hermen. einsetzt - so erhalten wir derngemäß einen Typus der zeitangebenden Worte, der sich zu den übrigen @́r, $\mu \alpha \sigma \iota$ völlig analog verhält, jedoch der Bestimmtheit seines Bedeutens durch die beigefügte Negaiionspartikel entkleidet ist. Darnach bezeichnet Aristoteles

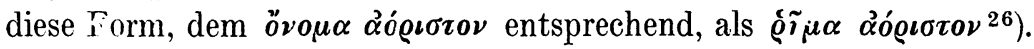
Später hingegen, wo das fertige Urteil auf die Beziehung von Bejahung und Verneinung hin untersucht wird, denkt er nirgends daran, die Form ,ou $\beta a \delta i \zeta \varepsilon \iota^{\prime \prime}$ als einheitlichen Prädikatsbegriff anzusprechen,

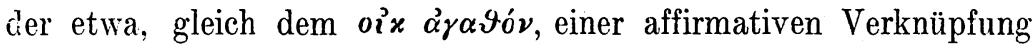

$\left.{ }^{24}\right)$ enthält es als ein logisches Moment; so jedoch, daß das $\mu \dot{\eta}$ غ $\varepsilon \bar{\nu} \nu \iota \iota$

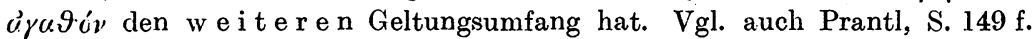
- Weiteres zum Verhältnis beider Formen s. u. S. $65 \mathrm{f}$.

${ }^{25}$ ) und, auf Grund des oben erwähnten Verhältnisses zwischen $\lambda \hat{\sigma} \gamma o \varsigma$

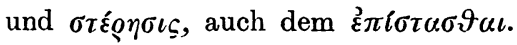

26) 3, 16 b $11 \mathrm{f}$. - Eine Form, die ein germanischer Sprachanalytiker niemals notiert hätte. Hier lag sie dadurch nahe, daß die Negationspartikel im Griechischen, wie auch in den romanischen Sprachen, sich sehr viel enger an das Verbum anlehnt als bei uns. 
mit dem Satzsubjekt fähig wäre $\left.{ }^{27}\right)$. Im Gegenteil vernachlässigt er

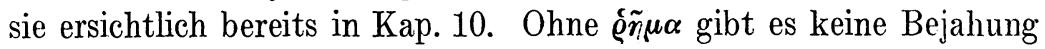
und keine Verneinung ( $19 \mathrm{~b} 10 \mathrm{ff}$; vgl. dagegen $16 \mathrm{~b}$ 12.) Hier ist also der bei der sprachlich-synthetischen Betrachtung der Sa t z-

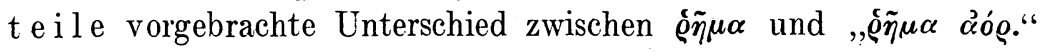
nunmehr für die logisch-analytische Betrachtung des S a t z e s selbst

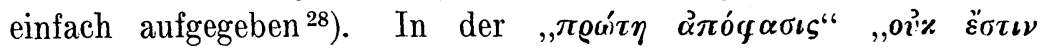

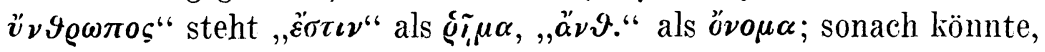
scheint es, für die verneinende Beurteilung dieses Inhalts (der

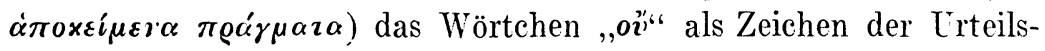
negation genügen; und man fragt sich, warum nicht dieses ron vornherein selbständig mit aufgeführt wurde, anstatt der ganzen merk-

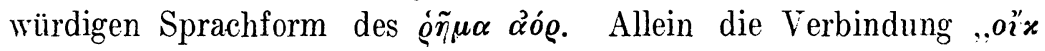
$\mathfrak{\varepsilon} \sigma \tau \iota \nu^{\prime \prime}$ erscheint doch auch wieder nach der logischen Seite als eine außerordentlich enge, wenn wir uns daran erinnern, daß eben das $\mu \dot{\eta}$ Eiv $\alpha \iota$ selber als der für das negative Urteil charakteristische b e u r teilende Z u sat z anzusehen ist. - Die Rede vom óruce

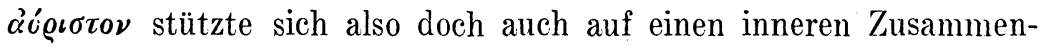
hang zwischen der Negation und irgendeiner Funktion des @ĩ $\tilde{q} \mu$, das wir daraufhin näher betrachten müssen.

Das @ॄ̃ $\mu \alpha$ wird (Kap. 3), abgesehen von seiner Abgrenzung gegen den $\lambda o ́ r o s$, nach zwei Seiten hin definiert. Erstens, im Anschluß an sprachlich Gegebenes: als ein Wort, in dessen Bildung ein Zeit-

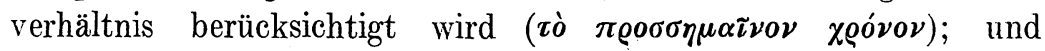
zweitens logisch: als ein Satzteil, der etwas von anderem Aus-

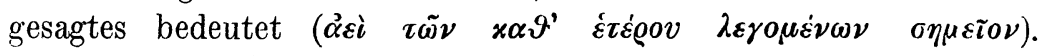
Das „Sein" aber - und sofern dieses in jedem enthalten, wiederum

$\left.{ }^{27}\right)$ Solche Verknüpfung wäre freilich denkbar. Da das $\beta u \delta i \zeta \varepsilon \iota$ so

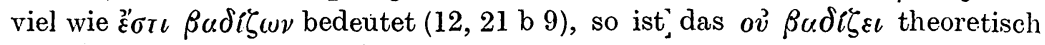
doppelt auflösbar: entweder in der Richtung der Satzverneinung $=$ oṽ

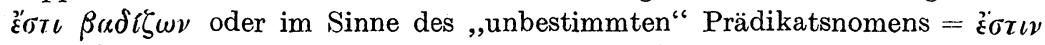
ov $\beta a \delta i \zeta \omega \nu$. Den letzteren Fall läßt Arist. unberücksichtigt, so daß die Auflösung jener Wortform in die Satzverneinung unvermeidlich ist.

${ }^{28}$ ) Schon Alexander hat sich in diesem Sinne geäußert (so nach Ammonius; - Ar. Op. ed. Acad. B. IV, p. 120 a 36 ff.). Innerhalb des Urteils

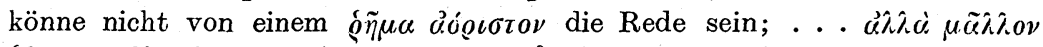

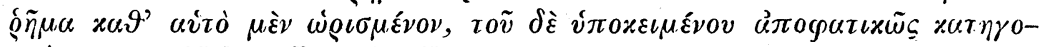

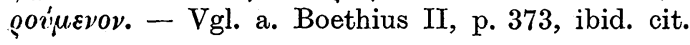


das $\varrho \tilde{\eta} \mu \alpha$ schlechthin - bedeutet außerdem eine Synthese, welche beide verbundene Teile voraussetzt, um gedacht zu werden ${ }^{29}$ ).

Daß mithin Aristoteles ,,die Copula noch nicht bestimmt vom Prädikat unterscheidet", muß $\mathrm{Z}$ ell e r ${ }^{30}$ ) insofern eingeräumt werden, als jener für unsere Begriffe „Copula“ und „Prädikat" wirk-

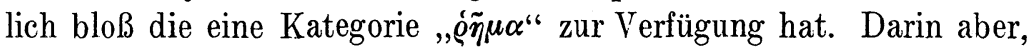
daß er ,die richtige Beziehung der Negation" ${ }^{31}$ ) im wesentlichen dennoch gefunden hat, zeigt sich gerade inmitten relativer Dürftigkeit des Begriffsmaterials die Zielsicherheit. seiner logischen Abstraktion.

Schon sprachlich stellt das Zeitwort, wie Aristoteles richtig fühlt, vermöge seiner Flexionsformen die unmittelbare Einheitsbeziehung innerhalb, des Satzes her ${ }^{32}$ ). - Deswegen ist es nicht bedeutungslos, daß sich das Negationszeichen gerade dann, wann es der Aufhebung jener $\mathrm{S}$ a t $\mathrm{z}$ einheit gilt, für das Sprachgefühl des Griechen aufs engste an das Zeitwort anschmiegt.

Logisch stellt das $\varrho \tilde{\imath} \tilde{\mu} \mu \alpha$ ein von anderem Ausgesagtes dar, - und ,, a u $ß$ e r d e m eine V e r k n ü p f u n g“". Offenbar wußte Aristoteles, als er jene Worte in $16 \mathrm{~b} 24$ schrieb, ziemlich genau, welche beiden Glieder in dieser Verknüpfung verknüpft würden. Er schickt voraus, daß die @́ $\eta_{\mu \alpha} \alpha \alpha$, wenn von ihrer Stellung im Urteil abgesehen wird,

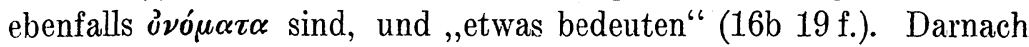
ergibt sich deutlich, wie hier für alle spätere Urteilsanalyse vorgearbeitet ist: Das $\varrho \check{r} r \mu \alpha$, sofern es ein Urteil herstellen hilft, bedeutet eine Synthese (Copula), und zwar zwischen einem viroxí-

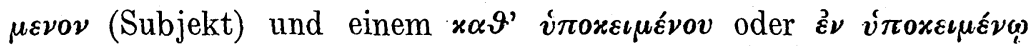
(16b 11) (Prädikat). Letzteres aber ist, ganz oder teilweise, im Sprachausdruck des @ं $\tilde{\mu} \alpha$ mitenthalten, sofern dieses, unter Absehung von jener Urteilsfunktion, ursprünglich gleichfalls ein o้vouc ist und ,etwas bedeutet".

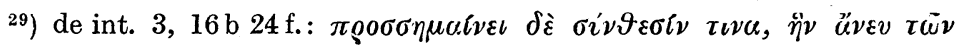

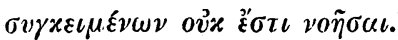

$\left.{ }^{30}\right)$ l. c. S. 221 . - Gegen Zellers, hiermit zusammenhängende Interpretation von de Int. 10, $19 \mathrm{~b} 19$ ff. vgl. Maier, S. 119, 1.

31) Entgegen Zellers Bemerkung, ebenda. - Vgl. Maier, S. 131, 4.

32) Und ,nur dadurch", nicht durch eine besondere Richtung seines

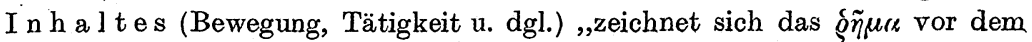
"vouce aus" (Steinthal S. 244). 


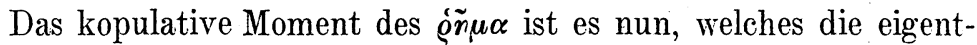
liche (widerspruchsweise) Satzverneinung sprachlich attrahiert, und welches logisch von ihr betroffen wird. - Aristoteles ist nicht dazu gelangt, jenes kopulative Moment rein herauszustellen; und demgemäß überhaupt nicht zu einem bestimmten Unterschiede zwischen

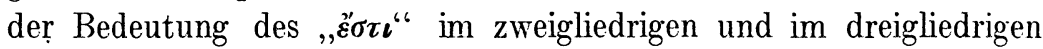
Satze $\left.{ }^{33}\right)$. - Daß aber das ,Sein" irgendwo l e d i g l i c h Verknüpfung bedeuten könne, kommt ihm gar nicht in den Sinn ${ }^{34}$ ). Dazu ist gleichsam die Nähe des Objektes viel zu groß. Es ist ein Hineinragen der Dinge selbst (sit venia verbo) in die Aussage, das aus dem Wahrsein der Synthese $\boldsymbol{A} \boldsymbol{B}$ in unmerklichem Übergange das reale Sein des Sachverhalts $\boldsymbol{A} B$ werden läßt.

Die Verbindung, welche das $\varrho \tilde{r}_{\mu} \mu$ mitbedeutet, bildet ein reales $\sigma v \gamma \approx \varepsilon \tilde{i} \sigma \vartheta a \iota$ nach. Die Trennung, welche sich ergibt, wenn das ov zum $\varrho \tilde{\eta} \mu \alpha$ tritt, muß ebenso einem realen $\delta \iota \eta \emptyset \tilde{\eta} \sigma \vartheta \alpha \iota$ entsprechen. - Allein das reale Getrenntsein ist für die Hermen. nicht mehr das Getrenntsein zweier realer Dinge. Denn nicht dem, was das övouce $\boldsymbol{A}$ und das $\varrho \tilde{r} \mu \alpha \boldsymbol{B}$ als solches ,,bedeutet", kommt Wahrheit und Wirklichkeit resp. Falschheit und Unwirklichkeit zu, sondern erst dem, was $\boldsymbol{A} B$ im Satze zusammen bedeuten (de Int. Kap. 1 u. 3). - Das Getrenntsein kann darum das objektive Korrelat wahrer (negativer) Sätze bilden, ohne die mindeste Garantie dafür, daß deren Substrat

33) Es handelt sich in Kap. 10, $19 \mathrm{~b} 15 \mathrm{ff}$.) um das Interesse an der rein formalen Möglichkeit, daß ein einfaches Urteil entweder aus zwei Teilen

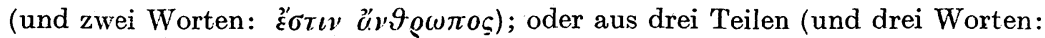

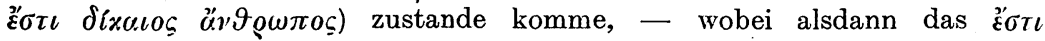

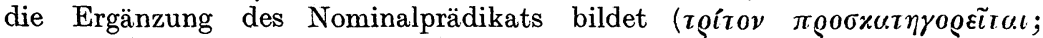
b 19). In diesem zweiten Falle ergibt sich, sofern das prädikative Nomen auch in ,unbestimmter" Form auftreten kann, die bekannte Doppelreihe der „Antithesen“. - Im Falle der Prädikation der übrigen @̊́ $\mu \alpha \tau \iota$ aber kennt Arist., wie wir sahen (s. o. Anm. 27), wieder nur eine Stellung der Ver-

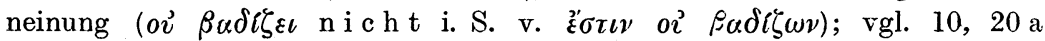
3-7. - Auch dies könnte uns darin bestärken, daß es in dem zweigliedrigen Satze (19 b 15) ,gleichgültig ist, welches Verbum zur Darstellung des Schemas verwendet werde“, (anders freilich Maier, S. 118 f.) und jedenfalls ,nicht an den sog. Existenzialsatz zu denken sei". So, völlig zutreffend, Prantl S. 147.

${ }^{34}$ ) Anders Waitz, S. 326, der mit Biese (Phil. d. Arist. I, 95, 3) aus 16 b $24 \mathrm{f}$. (vgl. dazu o. Anm. 9) herausliest, das $\varepsilon \tilde{\nu} \nu \alpha \iota$ habe nicht die Bedeutung eines $\oint \tilde{\eta} \mu \alpha$, weil .... es sich auf die Verbindung des Subjekts und Prädikats zu einem Ganzen bezieht". 
irgendwelche Gegenstände in der Welt bilden, denen mehr Sein zuläme, - als eben auch dem schlechthin Nichtexistierenden zukommt, wenn wir davon sagen: $\varepsilon^{\prime} \sigma \tau \iota ~ \delta o \xi \alpha \sigma \tau \tau^{\prime} \nu$. - Die Aufhebung der SatzSynthese $A B$ durch Satzverneinung bedeutet unmittelbar die Unwirklichkeit des fraglichen Sachverhalts, - wie die Bejạhung dessen Wirklichkeit -; sie sagt aber noch nichts über das M a B dieser Unwirklichkeit; sie kann auf ein faktisches Anders-Verbundensein der Urteilselemente zurückgehen, aber ebensowohl auch auf gänzliche Nichtexistenz des zum Subjekt und Träger der betr. begrifflichen oder akzidentellen Bestimmung gesetzten Dinges. Dann aber haben wir Liteile, von dadurch nicht beeinträchtigter Geltung, deren Substrate überhaupt nicht schlechthin, sondern bloß hypothetisch poniert werden; und diese Merkwürdigkeit finden wir gleich bei der Ein-

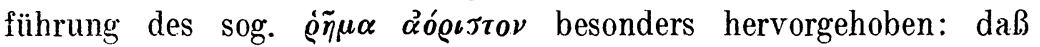
dieses jedwedem Substrat zukommen könne, gleichviel ob es existiere oder nicht. (3, $16 \mathrm{~b} 12 \mathrm{ff}$.)

Damit erst wird das Wesentliche des Unterschiedes zwischen dem formal bejahenden Satze: „, $\boldsymbol{A}$ ist Nicht- $\boldsymbol{B}$ ", und dem formal verneinenden:, $\boldsymbol{A}$ ist-nicht $\boldsymbol{B}^{\text {" }}$ erschöpft. Dieser gilt auch da, wo gar kein $\boldsymbol{A}$, welches $\boldsymbol{B}$ sein könnte, existiert; jener dagegen schließt die Wirklichkiet seines Substrates (i $\pi \boldsymbol{\pi} \boldsymbol{x} \dot{\boldsymbol{i}} \boldsymbol{\mu} \boldsymbol{\varepsilon} \boldsymbol{v}^{\prime} \boldsymbol{\nu} \boldsymbol{\nu}$ im Doppelsinne: Substanz-Subjekt) unbedingt ein, und vermag eben deshalb nicht, eine reine, dem Satze des ausgeschlossenen Dritten gemäße Antiphasis zu,$A$ ist $B^{\prime \prime}$ darzustellen:

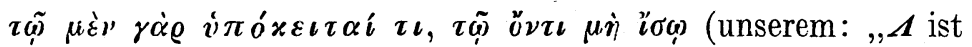

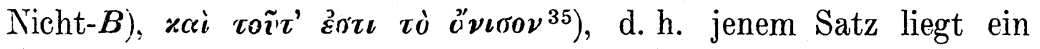
Existierendes $(A) \mathrm{zu}$ Grunde, welches die wirkliche Bestimmung aufweist, z. B. der Gleichheit beraubt zu sein.

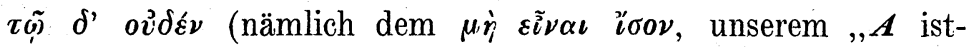
nicht $\left.B^{6}\right)$. D. h. bei reiner Satzverneinung braucht, unbeschadet der Wahrheit des Satzes, eine solches $\boldsymbol{A}$-keineswegs zu existieren ${ }^{36}$ ).

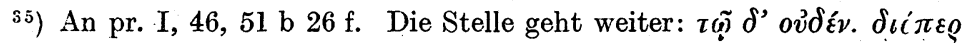

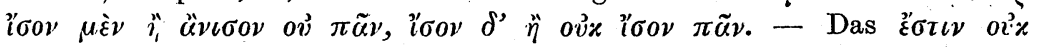

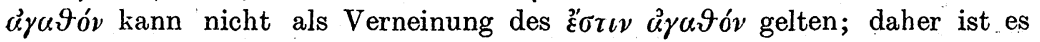

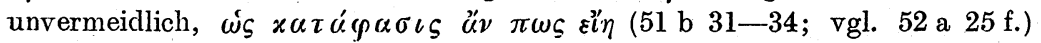

$\left.{ }^{36}\right)$ Unbeschadet auch der Geltung des Satzes vom ausgeschlossenen Dritten; existiert $A$ nicht, so ist die Bejahung , $A$ ist gut" stets falsch, die Verneinung , $A$ ist-nicht gut" aber stets wahr (vgl. a. Kat. 10, 13 b 27-33). 


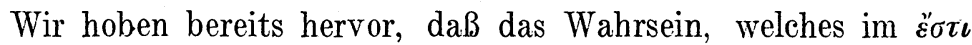
jederzeit latent ist, und das Wirklichsein, welches ein Satzsubstrat als existent bezeichnet, für Aristoteles ineinander übergehen. Wird nun aber die Synthesis zwischen $\boldsymbol{A}$ und $\boldsymbol{B}$, und das Wahrsein dieser Synthesis, beides ins Gegenteil verkehrt, sobald die Negation zum

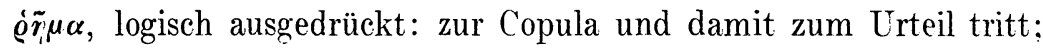
- so verhält es sich weniger einfach mit dem Wirklichsein, der Existenz. Es bleibt nicht, wie im Grunde bei Plato, die Existenz $\operatorname{des} \boldsymbol{A}$ ohne weiteres negierbar durch die Wahrheit von , $\boldsymbol{A}$ ist-nicht $\left.B^{: 637}\right)$. Nur k ö n n e n solche Sätze gelten auch von nicht existierendem A. - Anderseits wird in allen affirmativen (irgendwie ein ,.̌̈ $\sigma \tau \iota \nu$ enthaltenden) Sätzen der platonische Fortgang rom qualitativen zum modalen Sein vollzogen ${ }^{38}$ ), und dies gibt dann Sätzen, in denen die Verneinung bloß zum Prädikatsnomen getreten ist, also das ¿́r $\tilde{\mu}$ ce, selber nicht von ihr angegriffen, Existenzialität zu enthalten fortfährt, - den besagten Unterschied gegen die Satzverneinung.

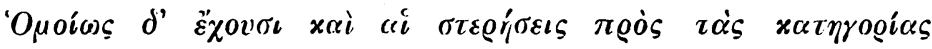

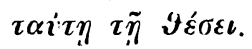

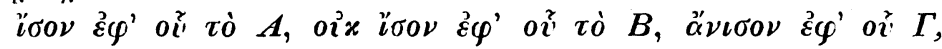

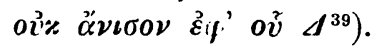

Wie die Sätze , $\boldsymbol{A}$ ist nicht-gleich" und , $\boldsymbol{A}$ ist-nicht nichtgleich", so verhalten sich zur einfachen Bejahung und Verneinung auch die Steresen; z. B. $\boldsymbol{A}$ ist ungleich, und $\boldsymbol{A}$ ist-nicht ungleich. Wir erkennen jetzt den Grund: Dem ,nicht-gleich“ als Prädikat eines Satzes, und dem ,ungleich“" als begrifflich-privativem Attribut

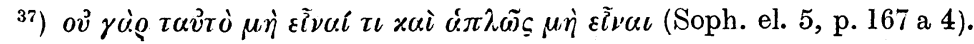

38) Daran kann nicht gezweifelt werden. Vgl. bes. An. pr. I, 46 a 29 f.:

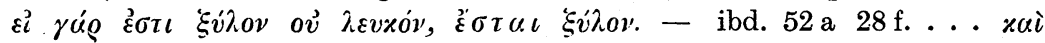

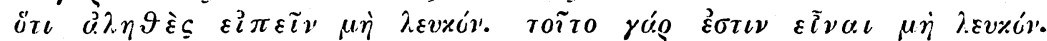
Auch wenn einmal ein Ansatz dazu gemacht wird, die Folgerung aus dem

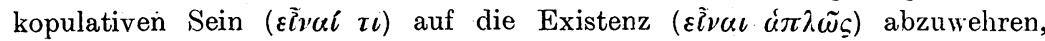
wie in de Int. 11, 21 a 25-28, so zeigt schon der Zusammenhang, daß hier kein klares Bewußtsein der Abwehr von Existenzialität als solcher aus dem हैं $\tau \iota \tau \iota, "$ besteht : nicht daß irgendwelches Existieren, sondern bloß daß das Schlechthin-Existieren - wozu das zur-Zeit-am-Leben-sein gehören würde - dem Homer abgesprochen werde, ist hier erfordert. Nichts anderes aber

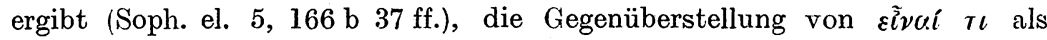

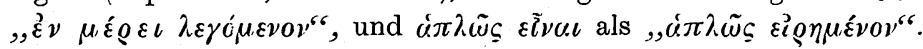

39) Vgl. die Tafel in unserem Text S. 208. - l. c. p. 52 a 15 ff.; - vgl. de Int. 10, 19 b 23 f. Hierzu Prantl, S. 150 Anm. 211. 


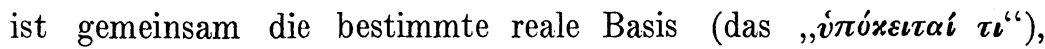
welche beide von reiner, formaler Negation trennt. Aristoteles glaubt, sich hiermit begnügen zu können, logisch aber ist damit freilich noch nicht das letzte Wort über diese Formen gesprochen. Denn

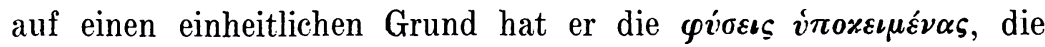

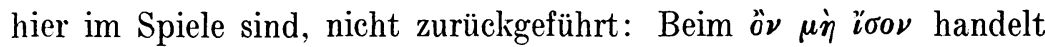
es sich um die $\mathrm{Ex}$ istenz eines Urteils subjektes $\boldsymbol{A}$. Beim

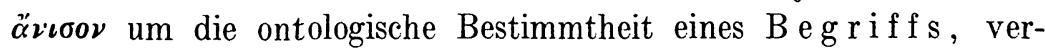
möge deren ihm das Attribut der Gleichheit entweder zukommen oder ,fehlen" (nie aber ohne Beziehung zu ihm sein) kann. - Wir finden hier den Privationsbegriff wiederum vermittelnd zwischen Gegensatz und Verneinung, wiederum nicht eben in der Richtung logischer Klarheit: Denn gewiß geht auch jetzt wieder der Gedanke des Geeignetseins für eine Bestimmung, resp. für eine bestimmte dichotomische Sphäre von Bestimmungen (z. B. die Handlungen als wesentlich geeignet für die Prädilkate sittlich oder nicht-sittlich) von dem

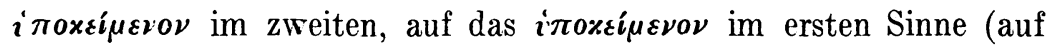

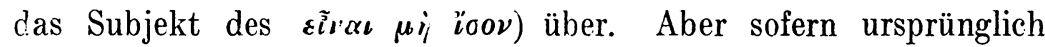
die bloße Setzung der Ex is te $\mathrm{nz}$ des $\boldsymbol{A}$ im formal-positiven Satze genügt, um das , $\boldsymbol{A}$ ist Nicht- $\boldsymbol{B}^{\prime \prime}$ vom , $\boldsymbol{A}$ ist-nicht $\boldsymbol{B}^{\text {" }}$ zu trennen, ist $\mathrm{Pr}$ a n $\mathrm{tl}$ schwerlich berechtigt, eine ,,die Gegensätze zugleich umfassende $\mathrm{P}$ ot e n $\mathrm{z}$ ", so wie beim Können und Wissen, nun auch ,, beim Sein" des Crteils , $\boldsymbol{A}$ ist $\boldsymbol{B}^{\text {" }}$ zugrunde zu legen $\left.{ }^{40}\right)$. -

Die Frage nach der logischen Koordination von Bejahung und Verneinung, welche in der neueren Logik stark diskutiert wird, ist auf dem aristotelischen Standpunkt noch nach keiner Seite eindeutig entschieden. Wie die Alternative von Wahrheit und Falschheit, von Verbunden- und Getrenntsein im Urteilsgebiete durchgeführt ist, - darnach scheint Aristoteles sich durchaus f ü $\mathrm{r}$ die Gleichordnung zu entscheiden. Allein wir sahen einmal, daß über die Sphäre des dianoetischen Denkens sich noch die des reinen Denkens, des voĩ im engeren Sinne, hinaushebt, welche diesen ganzen Dualismus nicht kennt. Und sodann, daß die erkenntnistheoretische Wertung des negativen Urteils als Konstatierung einer objektiven Diärese auch diesem eine positive Färbung gibt.

40) Prantl gewinnt dadurch den inneren Grund, warum Arist. ,,in die Lehre vom Urteil den Begriff des Möglichen beiziehen mußte" (S. 151 ff.; vgl. aber die Restriktion jener Behauptung, die er selbst S. 159 f. vornimmt). 
Die Suprematie der B e ja h u ng war in jeder auf Konstruktion und Vergleichung von bloßen Begriffen abgestellten Logik unvermeidlich. Irrtum und Verneinung werden der platonischen Ideenlehre gegenüber zu einem Probleme, das sie in ihrem eigenen Rahmen nicht völlig lösen kann. Das Problem, durch Platos „Sophistes" eher entfaltet als gelöst, gipfelt in der Schwierigkeit rom „Sein des Nichtseienden".

Aristoteles hat die Akademie nicht mit einer fertigen Urteilslehre verlassen. Ursprünglich ist vielmehr auch bei ihm das Urteil noch nicht für sich klar herausgestellt. Sätze, die auf Wahrheit vollen Anspruch machen können, sind zunächst solche, deren Prä-

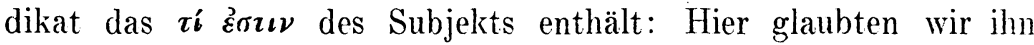
noch auf einer Stufe der Utberordnung der Affirmation zu beobachten. Iie Alternative von wahr und falsch ist in der Kateg.-Schrift als bekannt rerzeichnet, aber noch nicht tiefer begründet. - Dies ändert sich später, da die Syllogistik ihn zu einer formaleren Betrachtung. des Urteils drängt.

Die Aporien vom ,Sein des Nichtseins" werden gründlichst in Angriff genommen und finden ihre Lösung in der Unterscheidung verschiedener Bedeutungen des Seins und Nichtseins. Zunächst in der Kategorienlehre als Basis aller anderen Distinktionen - wobei

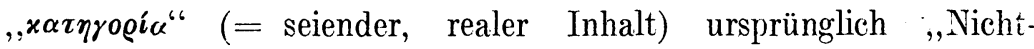
sein" ausschließt ${ }^{41}$ ) - später in den Parallelismus des affirmativen und negativen Aussagens einbezogen wird.

Dann in der Unterscheidung aktuellen und potenziellen, ferner wesentlichen und akzidentellen Seins ${ }^{42}$ ). Mit letzterer Distinktion wird die Lösung der Urteilsaporien ganz ähnlich gefördert wie mit ersterer die der Rätsel des Werdens. - Wie ist es möglich, daß aus einem $\boldsymbol{A}$ dessen Gegenteil $\boldsymbol{B}$, ,werde“? Entweder $\boldsymbol{B}$ war vorher schlechtweg nicht da; dann ist unerklärlich, wie es entstand. Oder es war da, dann waren beide Gegenteile zugleich da, was unmöglich scheint. Mit der Einführung des potenziellen Seines ergibt sich, daß $\boldsymbol{B}$ zwar

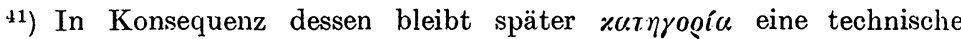

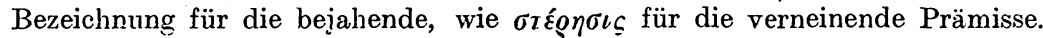

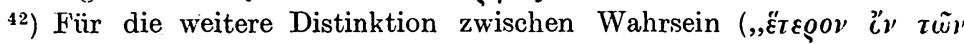

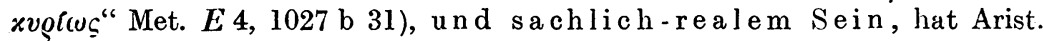
ebenfalls eine gewisse Vorliebe gezeigt, aber wahrlich eine in jedem Sinne platonische. (Dazu Met. $\Theta$ 10.) 
da war, nämlich $\delta v \nu$ ó $\mu \varepsilon \iota$ oैv; daß aber dieses Dasein, als ein bloß potenzielles, keineswegs ein aktuelles Zugleichsein der Gegenteile involviert (Met. $\Gamma 51009 \mathrm{a} 35$ f.; vgl. Met. $\boldsymbol{A} 2$; Phys. I, 8). Ähnlich besteht im Urteil der scheinbare Widerspruch ,,s. ist klug." und zugleich: ,s." ist nicht ,,klug". Die Einführung des akzidentellen Seins entscheidet nun, - während Plato den gordischen Knoten zerhieb -: ,s. ist klug“" bleibt wahr, sofern das Klugsein dem s. (akzidentell) zukommt; — ,s.“ ist nicht ,klug" bleibt nur insofern ebenfalls wahr, als das akzidentelle Klugsein eben keineswegs das ,,begrifflich, wesentlich Klugsein" involviert.

Von dem spezifisch begrifflichen Verhältnis zwischen den Sub-

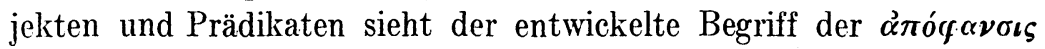
grundsätzlich $a b^{43}$ ). Er geht auf akzidentelle so gut wie auf essenzielle Aussagen, und der schwächere Teil ist nun entscheidend: Insoweit es der Akzidenz von vornherein eigen ist, daß sie einem Substrat sowohl zukommen als auch nicht-zukommen, und demgemäß das Urteil über das Verknüpftsein beider wahr-oder-falsch sein kann, ist jetzt auch eine bewußte logische Koordination von Bejahung und Verneinung vollzogen.

Der Unterschied von essenziellem und akzidentellem Sein ist nicht zu einem Gegensatz beider gesteigert. Vielmehr bleibt im Urteil immer ncoh ein Rest heimlicher Herrschaft sozusagen des Subjekts als des Wesensbegriffs: auch dem akzidentellen Prädikat gegenüber wird das Subjekt unter Umständen so behandelt, a ls

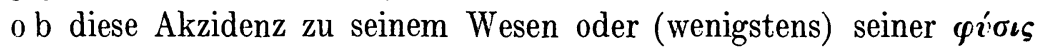
gehöre: Dann besteht für das Subjekt die Alternative eines Habens

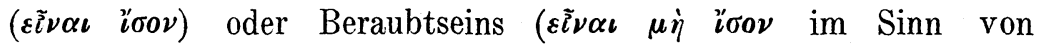

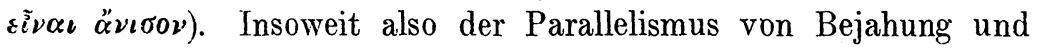
Verneinung bei Aristoteles zu dem eigentümlichen Bègriffe der Privation führt, hat er selber im Grunde eine über die bloß psychologische Synthesis hinausgehende positive Beziehung zwischen Subjekt und Prädikat in allen Urteilen zu seiner Voraussetzung.

43) Daß er auch von den Begriffen der Notwendigkeit und Möglichkeit nach deren metaphysischer Richtung hin absieht, hat Maier gezeigt. 